\title{
Laparo-Endoscopic Single Site Retroperitoneal Partial Nephrectomy: A Novel Concept
}

\author{
Alejandro R. Rodriguez, Tariq Hakky, Philippe E. Spiess \\ Department of Urology, Moffitt Cancer Center and Research Institute and University of South Florida, \\ Tampa, Florida
}

\begin{abstract}
Purpose: Various treatment options are available for small incidentally detected kidney masses, including surveillance, partial nephrectomy and probe ablative therapies. When partial nephrectomy is considered, the procedure can be safely approached laparoscopically, either pure or robot assisted, in experienced hands. Laparo-endoscopic single site (LESS) surgery is a novel approach for partial nephrectomies in well selected cases. In this video, we present our experience with the LESS retroperitoneal partial nephrectomy using the Gelpoint device.

Material and Methods: A 63 year old male patient with a BMI of 31, and a history of a T1c prostate cancer, had a $1.5 \mathrm{~cm}$ right posterior lower pole renal enhancing mass discovered incidentally on a three phase CT scan. With the patient under general anesthesia, and in a full flank position, a LESS retroperitoneal partial nephrectomy was performed using a $3 \mathrm{~cm}$ transverse incision below the tip of the 12 th rib. The following instruments and devices were used: A gelpoint device for single incision port of entry, one $10 \mathrm{~mm}$ and two $5 \mathrm{~mm}$ trocars used through the gelpoint, one $5 \mathrm{~mm}$ Olympus HD endoeye flexible tip camera, one roticulator scissors, and one articulating graspers.

Results: Operative time, EBL, and hospital stay were 1 hour, $5 \mathrm{ml}$, and 23 hours, respectively. The pathology result confirmed a benign hemorrhagic cystic mass. The visual analog scale (0-10) for pain at recovery, 6 hours post op, and 23 hours post op was 5, 3, and 1 point, respectively. The patient tolerated clear liquids and regular diet at 6 and 16 hours, after the procedure. At one month of follow-up, the patient is asymptomatic and practically scarless.

Conclusions: LESS retroperitoneal partial nephrectomy is safe and feasible in selected cases such as small exophytic posterior renal masses. The retroperitoneal approach avoids mobilization of the colon and kidney to access the posterior surface. Instrument clashing, limited range of motion, and CO2 leakage, can be some difficulties encountered during single port retroperitoneal surgery. However, the Gelpoint device gives a great seal in the flank position and allows the relocation of trocars, without loss of $\mathrm{CO} 2$ pressure, to prevent instrument clashing during different parts of the procedure.
\end{abstract}

Int Braz J Urol. 2011; 37 (Video \#2): 136_7

Available at: www.brazjurol.com.br/videos/january_february_2011/Rodriguez_136_137video.htm

\section{Correspondence address:}

Dr. Alejandro R. Rodriguez

Department of Urology

Moffitt Cancer Center

8203 Whistling Pine Way

Tampa, FL, 33647, USA

E-mail: armbkdd@yahoo.com 


\section{EDITORIAL COMMENT}

In this novel video by Rodriguez and colleagues, a novel approach for a small exophytic renal lesion is presented. Since the first laparoscopic single site surgery (LESS) report in the urological literature by Rane in 2007 , different urological procedures including pyeloplasty, renal cryotherapy, adrenalectomy, varicocelectomy, ureterolithotomy have been described (1). This approach uses a single surgical access following the same general principles of pure laparoscopic surgery, with its inherent benefit of minimal surgical trauma. Previously, White et al. described eight LESS retroperitoneal procedures including one partial nephrectomy (2). Aaron et al. reported 5 partial nephrectomy using a transperitoneal (trans-umbilical) LESS approach using a $2 \mathrm{~mm}$ port in the axillary line (hybrid technique) (3). Recently, Cindolo et al. reported six LESS cases using a transperitoneal approach, all performed without hilar clamping, one of which required conversion to a standard laparoscopic approach to control bleeding and two necessitating an extra port for renal parenchyma suturing and liver retraction (4).

Single port surgery is considered minimally invasive laparoscopy, and is an alternative to standard laparoscopy in appropriately selected cases. It however remains a technically challenging procedure even for experienced laparoscopists. Instruments for LESS are rapidly evolving in order to avoid clashing and compensate for their limited range of motion. In this regard, robotic technology is trying to eliminate these current limitations.
It remains that partial nephrectomy is a very challenging procedure when a minimally invasive approach is chosen. In this regard, it is imperative to have careful patient selection when a new technique such as this is employed. Carefully chosen cases such as the one shown in the present video by Rodriguez et al. can translate into excellent peri- and postoperative outcomes. Future randomized trials comparing pure laparoscopic and LESS surgery will help delineate the role and merit of this technique. At the present time, careful patient selection remains the key factor predicting a favorable outcome with these novel new surgical techniques to the small enhancing renal mass.

\section{REFERENCES}

1. Rane A, Rao P, Bonadio F, Rao P: Single port laparoscopic nephrectomy using a novel laparoscopic port (R-port) and evolution of single laparoscopic port procedure (SLIPP). J Endourol. 2007: 21 (Suppl 1): A287, V18-11.

2. White WM, Goel RK, Kaouk JH: Single-port laparoscopic retroperitoneal surgery:initial operative experience and comparative outcomes. Urology. 2009; 73: 1279-82.

3. Aron M, Canes D, Desai MM, Haber GP, Kaouk JH, Gill IS: Transumbilical single-port laparoscopic partial nephrectomy. BJU Int. 2009; 103: 516-21.

4. Cindolo L, Berardinelli F, Gidaro S, Schips L: Laparoendoscopic single-site partial nephrectomy without ischemia. J Endourol. 2010; 24: 1997-2002.

Dr. Jose Jaime Correa Urologic Oncology Department Hospital Pablo Tobon Uribe Medellin, Colombia E-mail: jocorreao@uces.edu.co 